\title{
Diffuse-object holograms in silver halide emulsions: influence of the beam ratio on the efficiency and the signal-to-noise ratio
}

\author{
A. Fimia, R. Fuentes, and A. Beléndez
}

\begin{abstract}
The influence of the beam ratio between reference and object beam intensities on the characteristics of diffuse-object holograms recorded as volume phase holograms in bleached silver halide emulsion is experimentally analyzed. Measurements of the diffraction efficiency and the signal-to-noise ratio of the holograms are taken. The experimental results presented show that when the beam ratio increases, the diffraction efficiency decreases and the signal-to-noise ratio increases; these two holographic parameters behave in this way no matter what type of processing is used.

Key words: Holographic recording materials, silver halide emulsions, diffuse-object holograms. (C) 1996 Optical Society of America
\end{abstract}

\section{Introduction}

Nowadays, holography is increasingly important in different fields of optical technology, and many types of holograms are now being used for scientific and technical applications. For example, holograms are among the most important components for some optical systems: lenses, mirrors, couplers between optical fibers, couplers between waveguides, optical fiber demultiplexers, optical memories, and optical interconnects for computing are representative devices that have been proposed and fabricated with some success. A feature of all these elements is that they transform input wave fronts into output wave fronts. In order to obtain these holographic optical elements with high diffraction efficiency, it is necessary to record these elements as volume phase holograms. ${ }^{1}$ Photographic emulsions are an important medium for making these volume holograms because of the relatively high sensitivity and ease of processing of the material, improved processing chemistries, and repeatibility of the results.

A. Fimia and R. Fuentes are with the Laboratorio de Optica, Departamento Interuniversitario de Optica, Universidad de Alicante, Apartado 99, Alicante E-03080, Spain. A. Beléndez is with the Departamento de Ingeniería de Sistemas y Comunicaciones, Universidad de Alicante, Apartado 99, E-03080 Alicante, Spain.

Received 5 July 1995; revised manuscript received 12 September 1995.

0003-6935/96/050782-05\$06.00/0

(C) 1996 Optical Society of America
Volume phase holograms are attractive for use as optical elements or for holographic storage owing to their high potential efficiency and high information densities. One of the most common materials is photographic emulsion. Volume holograms recorded in silver halide emulsions are commonly bleached to obtain phase holograms in order to increase diffraction efficiency. ${ }^{2-5}$ In general, diffraction efficiency is a function of the recording material and the processing chemistry ${ }^{6-8}$ as well as of the exposure or the balance ratio between reference and object beam intensities. Unfortunately, although the resulting phase holograms have high diffraction efficiency, this is usually accompanied by an increase in the scattering of light from the silver halide grains $\mathrm{s}^{9,10}$ and a consequent reduction in image quality. Scattering is due to the granular structure of the silver halide material and takes place both at the recording and at the reconstruction stage.

The properties of diffuse-object holograms recorded in photographic emulsion have been amply studied. From a theoretical point of view, the study done by Upatnieks and Leonard ${ }^{11,12}$ offered the first theoretical model, and this model, together with the one presented by Kogelnik ${ }^{13}$ for holographic gratings, made it possible to analyze and study noise in diffuse-object holograms. This model proved the importance that the beam ratio has in the signal-tonoise ratio of a diffuse object. More recent studies ${ }^{14,15}$ have shown that noise gratings are one of the many sources of noise in the image of diffuse-object holograms. 
In this paper we experimentally analyze the influence of the balance ratio between reference and object beam intensities on the diffraction efficiency and the signal-to-noise ratio of diffuse-objects holograms recorded in bleached silver halide emulsion. We consider rehalogenating bleaches with and without a fixation step.

\section{Experimental Procedure}

Diffuse-object holograms were recorded on AgfaGevaert 8E75 HD emulsion, and the exposures were made with $632.8-\mathrm{nm}$ radiation from a $\mathrm{He}-\mathrm{Ne}$ laser. The object used was a $2 \mathrm{~cm} \times 2 \mathrm{~cm}$ square with a central opaque zone measuring $1 \mathrm{~cm} \times 1 \mathrm{~cm}$. The reference beam was collimated, and it was polarized perpendicular to the plane of incidence. The distance of the object from the recording medium was $30 \mathrm{~cm}$, and the reference beam formed a $37.5^{\circ}$ angle with the normal of the holographic plate, which was parallel to the object. Figure 1 shows a schematic representation of the geometry used in our experiments. The reference-to-object beam ratios considered were $k$ equal to $1.5,3.5,9,18$, and 40 .

Twelve holograms were made with exposures in the range from 10 to $340 \mu \mathrm{J} / \mathrm{cm}^{2}$. Spurious reflections were eliminated by placement of an indexmatched absorbing layer against the glass side of the photographic plate. After exposure, the plates were developed in PAAAC developer. ${ }^{16}$ The developed plates were rinsed briefly and bleached without a fixation step. Two types of bleach baths were used in these experiments: one was R-10, and the other was EDTA. Both of these are rehalogenating bleach baths. In these rehalogenating bleaches, the bleach baths contain a rehalogenating agent (potassium bromide) that converts most of the developed silver back into a silver halide. In this case a phase hologram is obtained mainly by the transfer of silver halide between the unexposed and the exposed areas. ${ }^{17,18}$ These bleaches have the advantage that the resulting emulsion thickness change produced by these baths is very small $(<0.05 \mu \mathrm{m})$ in the nominally thick 6 - $\mu \mathrm{m}$ film, ${ }^{19,20}$ since the overall

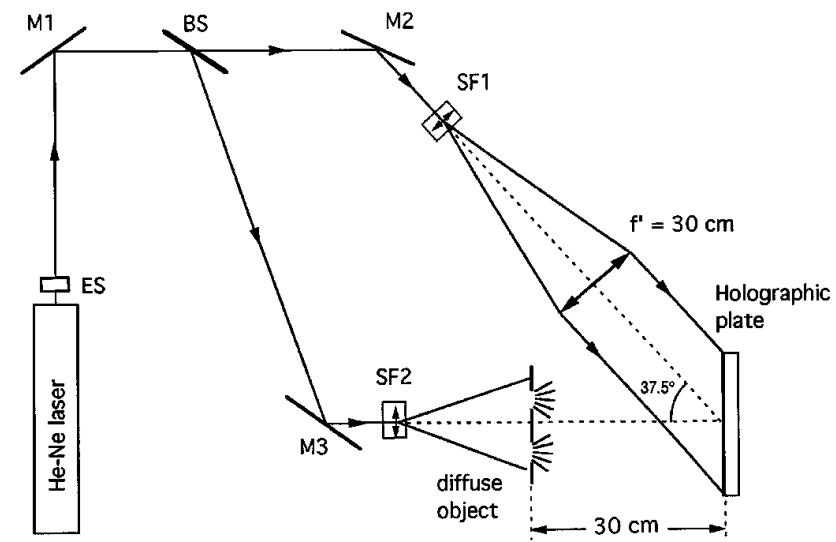

Fig. 1. Experimental setup for recording diffuse-object holograms: BS, beam splitter; ES, electronic shutter; M1-M3, mirrors; SF1, SF2, spatial filters.
Table 1. Processing Schedule for Holograms: Fixation Step Not Used in Holograms

\begin{tabular}{lc}
\hline \multicolumn{1}{c}{ Step Procedure $^{a}$} & Time $(\min )$ \\
\hline 1. Develop (PAAAC) & 3 \\
2. Rinse in running water & 1 \\
3. Bleach (R-10 or EDTA) & $\sim 3$ \\
4. Wash in running water & 5 \\
\hline
\end{tabular}

${ }^{a}$ All solutions at $20^{\circ} \mathrm{C}$.

removal of silver salts from the emulsion is minimal. Also it is assumed that the average refractive index does not change appreciably as a result of processing. ${ }^{19,20}$ As thickness and the average refractive index of the holographic recording material show very little change when this type of chemical processing is used, the reconstruction geometry of the holograms corresponding to maximum diffraction efficiency coincide with the construction geometry if recording and readout wavelengths are equal. This implies that Bragg's law is complied with in the reconstruction stage, although sometimes there is a displacement of the Bragg angle that is caused by shear-type effects. ${ }^{21,22}$ The results obtained when no fixation step was used are compared with the results obtained for diffuse-object holograms for which a fixation step was used in processing. The latter holograms were obtained with a process in which the emulsion was developed, fixed, and then bleached. This is known as a rehalogenation process; as a part of the process, the exposed areas in which there are traces of metallic silver are subsequently processed, and as a result they once again have silver halide in them. This generates a clear difference in the refractive index of the silver halide gelatin area and the area in which there is only gelatin.

Details of the processing schedule as well as the developer and the bleach bath formulas are given in Tables 1-3.

\section{Experimental Results}

The holograms were replayed in air with the conjugate of the collimated reference wave, and the diffracted output beam formed the real image of the object. The diffraction efficiency and the signal-tonoise ratio $(\mathrm{SNR})$ were measured as a function of the replay angle with the same wavelength that was

Table 2. Processing Schedule for Holograms: Fixation Step Used in Holograms

\begin{tabular}{lc}
\hline \multicolumn{1}{c}{ Step Procedure $^{a}$} & Time $(\mathrm{min})$ \\
\hline 1. Develop (PAAAC) & 3 \\
2. Rinse in running water & 1 \\
3. Fix with F-24 & 4 \\
4. Wash & 10 \\
5. Bleach (ferricyanide) & 10 \\
6. Wash in running water & 5 \\
\hline
\end{tabular}

${ }^{a}$ All solutions at $20^{\circ} \mathrm{C}$. 
Table 3. Developer and Bleach Bath Formulas
PAAAC developer

Sodium carbonate

Ascorbic acid

Phenidon

Distilled water to make $1 \mathrm{~L}$

R-10 rehalogenating bleach bath

Potassium dichromate

Sulfuric acid

Potassium bromide

Distilled water to make $1 \mathrm{~L}$

EDTA rehalogenating bleach bath

Potassium bromide

Sulfuric acid

Distilled water to make $1 \mathrm{~L}$

Ferricyanide rehalogenating bleach bath

Potassium ferricyanide

Potassium bromide

Distilled water to make $1 \mathrm{~L}$
Ferric sulfate
$120 \mathrm{~g}$

$18 \mathrm{~g}$

$0.5 \mathrm{~g}$

$2 \mathrm{~g}$

$10 \mathrm{~mL}$

$35 \mathrm{~g}$

$30 \mathrm{~g}$

$30 \mathrm{~g}$

$10 \mathrm{~mL}$

$7 \mathrm{~g}$

$8 \mathrm{~g}$

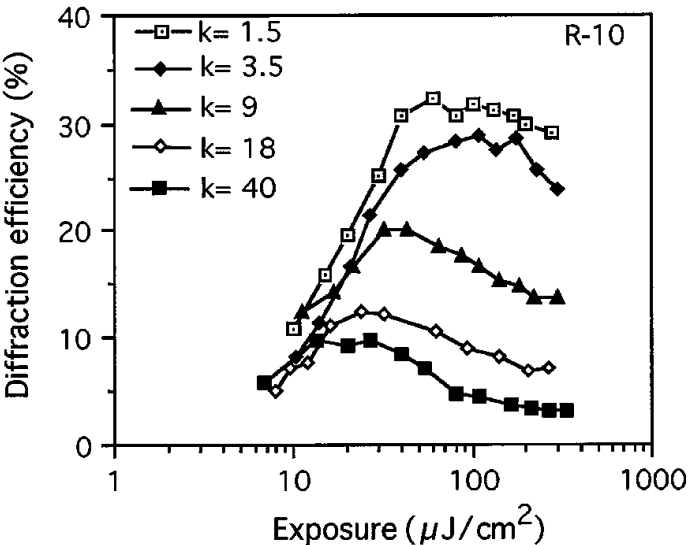

Fig. 3. Same as Fig. 2 except that the plates were bleached with R-10 (see Table 1).

object and the incident light intensity $I_{i}$ :

$$
N=\frac{I_{0}}{I_{i}}
$$

used during the recording stage. The SNR was defined as the relation between the value of the light intensity on each side of the square, $I_{1}$ and $I_{2}$, divided by the value of the light measured in the center of the square $I_{0}$ in the central square of the reconstructed object:

$$
\mathrm{SNR}=\frac{I_{1}+I_{2}}{I_{0}}
$$

Therefore the SNR gives us the contrast at the reconstructed object. In all cases the experimental measurements for the intensities were corrected taking into account losses that were due to reflection at the two surfaces of the plates. SNR values of 1 are considered very noisy, and values of 60 or more indicate that, as might be expected, practically no light reaches the central zone of the square. The noise $N$ was measured as the ratio between the light intensity $I_{0}$ in the central square of the reconstructed

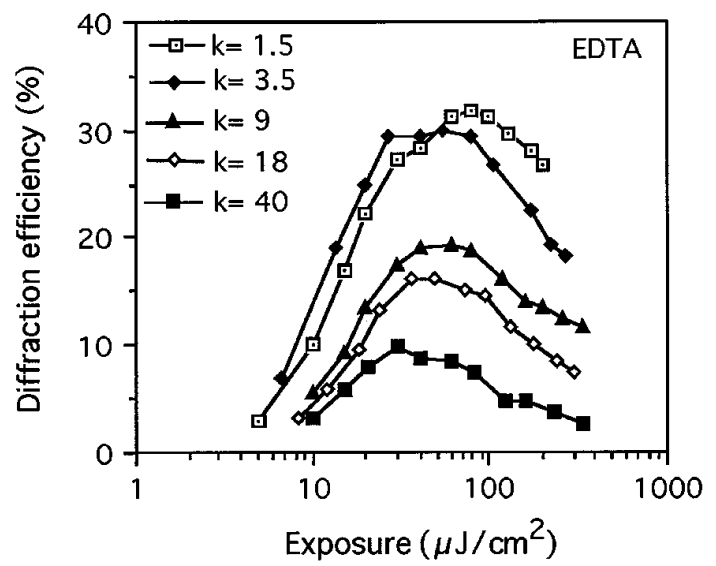

Fig. 2. Comparison of diffraction efficiency as a function of exposure for different beam ratios when reconstruction is done at the Bragg angle. Plates were processed without a fixation step and bleached with EDTA(see Table 1).
Taking diffraction efficiency and SNR as the parameters for study, we analyzed how these varied in relation to exposure when the beam ratio was modified. The diffraction efficiency as a function of exposure for the processing used in our experiments is presented in Figs. 2-4. As we can see in these figures, when the beam ratio increases, the diffraction efficiency decreases. However, when the beam ratio increases, the SNR increases, as we can see in Figs. 5-7, in which we present the SNR as a function of exposure for the processing used. These two functions, diffraction efficiency and SNR, behave in this way no matter what type of processing is used.

As regards the types of processing that produced noise gratings, we showed that the efficiency of these noise gratings $\Delta I_{n}$ (which was defined in Ref. 14 as the difference between the peak diffraction efficiency and the efficiency at the reference angle) decreases with the beam ratio. Thus we see in Fig. 8 that when the beam ratio $k$ goes from 1.5 to 9 , the

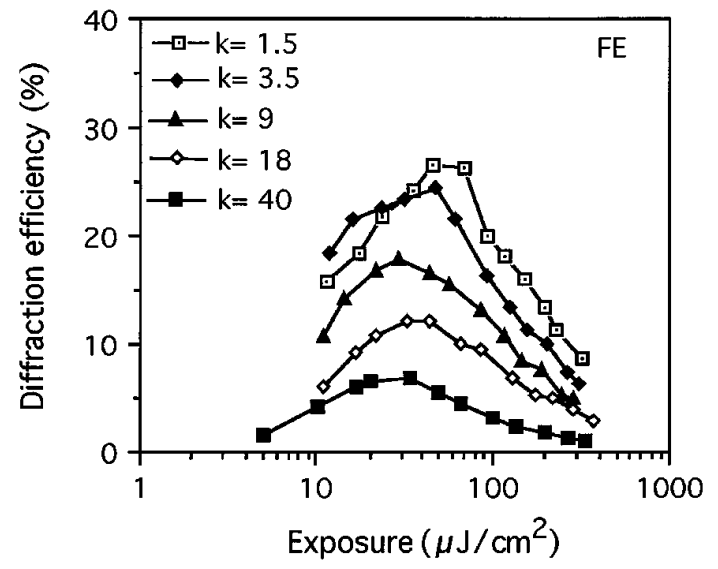

Fig. 4. Same as Fig. 2 except that the plates were processed with a fixation step and bleached with a ferricyanide bleach bath (see Table 2). 


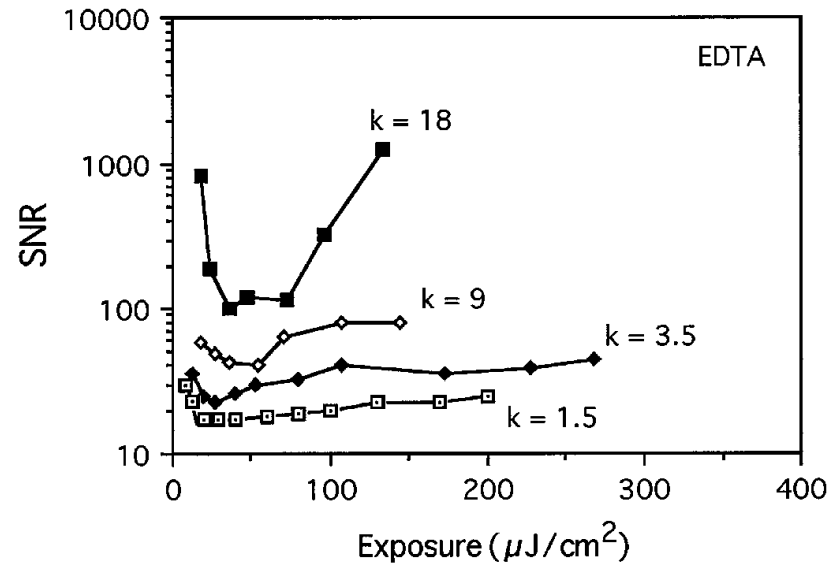

Fig. 5. Comparison of the SNR as a function of exposure for different beam ratios when reconstruction is done at the Bragg angle. Plates were processed without a fixation step and bleached with EDTA(see Table 1).

minimum that appears in the diffraction efficiency curve when reconstruction is done with the Bragg angle decreases even more. As a matter of fact, when $k=1.5, \Delta I_{n}=5.8 \%$, and when $k=9, \Delta I_{n}=$ $4.6 \%$. However, the effect of noise gratings on efficiency is more important for high values of $k$ because the maximum expected efficiency, $\eta_{\max }$, is higher for lower values of $k$. For example, for $k=1.5, \eta_{\max }$ is approximately $34 \%$ and $\Delta I_{n}=5.8 \%$. This implies that $\Delta I_{n} / \eta_{\max } \approx 0.17$. On the other hand, for $k=9$, we have $\eta_{\max } \approx 21 \%, \Delta I_{n}=4.6 \%$, and then $\Delta I_{n} / \eta_{\max } \approx$ 0.22 .

Another fact that reconfirms the claim that an equivalent grating can be assigned to the noise function is that the grating reacts to the beam ratio in exactly the same way as the diffraction efficiency of the main grating does. A similar definition to the definition of $\Delta I_{n}$ is considered for analysis of the drop in the noise that is due to noise gratings $\Delta N$. In other words, when the beam ratio increases, the noise grating's influence on total noise $\Delta N$ also increases and therefore the minimum noise that is obtained in the Bragg angle also decreases when the

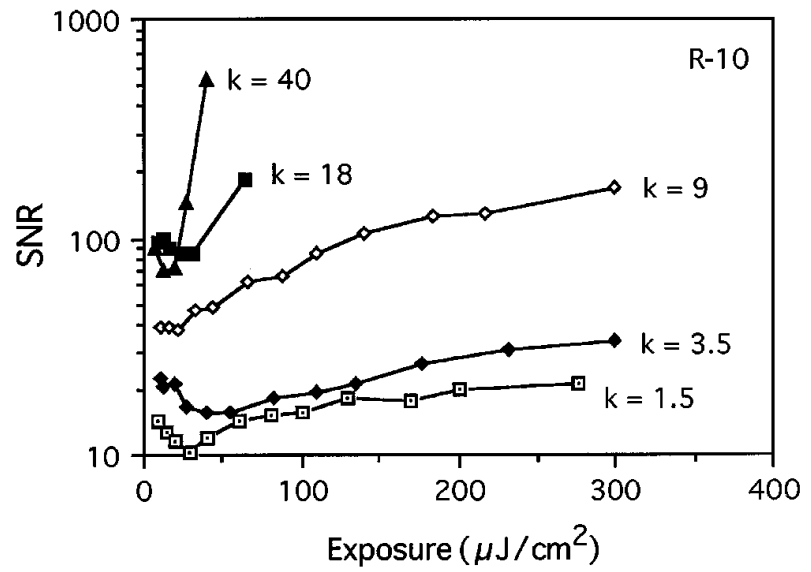

Fig. 6. Same as Fig. 5 except that the plates were bleached with R-10 (see Table 1).

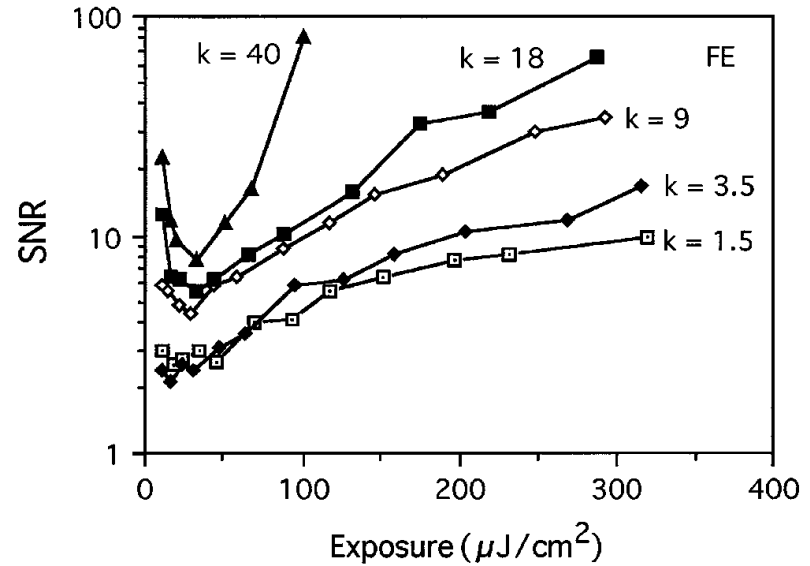

Fig. 7. Same as Fig. 5 except that the plates were processed with a fixation step and bleached with a ferricyanide bleach bath (see Table 2).

beam ratio increases (see Fig. 9). In this case, when $k=1.5, \Delta N=0.19 \%$, and when $k=9, \Delta N=0.11 \%$. The effect of noise gratings on noise is more important for high values of $k$ because the maximum expected noise $N_{\max }$ is higher for lower values of $k$. For example, for $k=1.5, N_{\max }$ is approximately $1.25 \%$ and $\Delta N=0.19 \%$. This implies that $\Delta N / N_{\max }$ $\approx 0.15$. However, for $k=9$, we have $N_{\max } \approx 0.20 \%$, $\Delta N=0.11 \%$, and then $\Delta N / N_{\max } \approx 0.55$.

This effect of the beam ratio on the characteristics of noise gratings in diffuse-object holograms occurs because for beam ratios greater than unity, the desired diffuse-object pattern is recorded at a weaker level, but there is one relatively strong of set noise gratings. The reconstructed object is now much dimmer compared with the recreated noise, and the SNR may be much worse. ${ }^{23}$ The influence of the beam ratio on noise gratings observed in diffuseobject holograms shows that the scatter gratings dominates the main desired diffuse-object hologram, and the effect is similar to the effects observed when holographic gratings are stored..$^{24}$

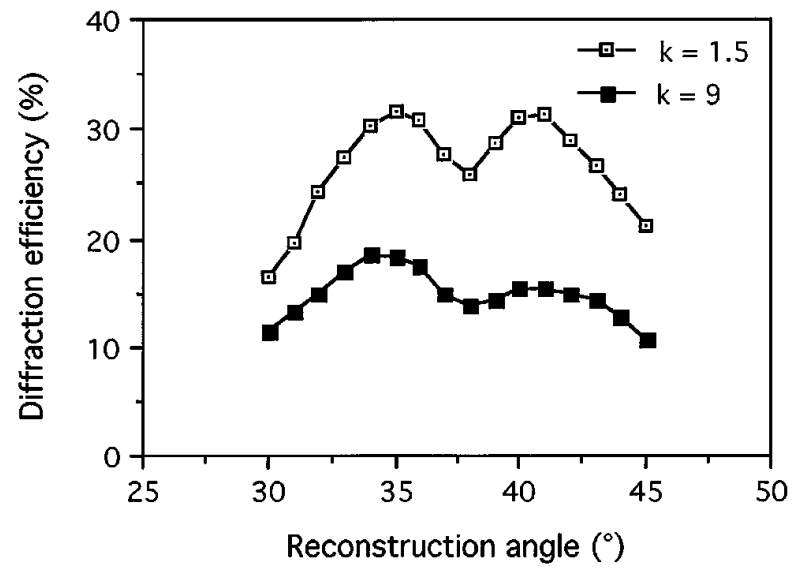

Fig. 8. Diffraction efficiency as a function of the reconstruction angle when the beam ratio varies. The plates were processed without a fixation step and bleached with EDTA. Exposure was $100 \mu \mathrm{J} / \mathrm{cm}^{2}$. 


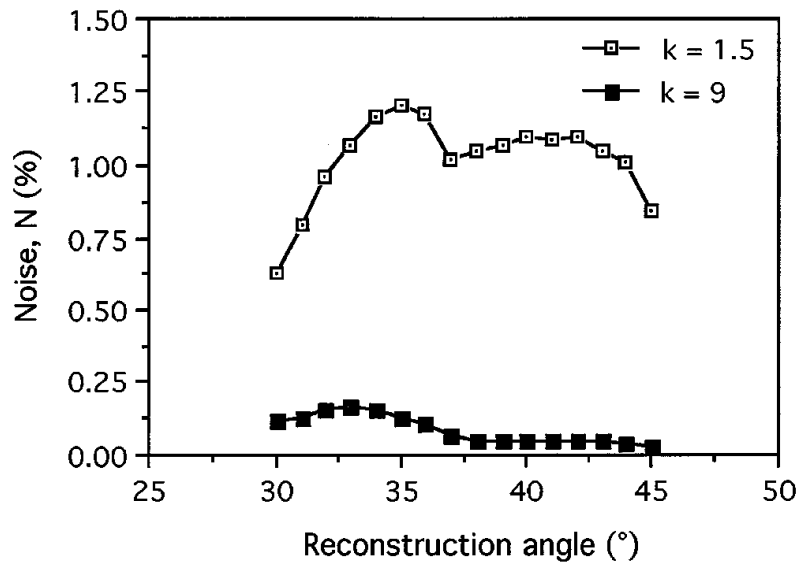

Fig. 9. Same as Fig. 8, but for noise efficiency $N$.

\section{Conclusions}

In summary, the experimental results shown in this paper indicate that the diffraction efficiency and the signal-to-noise ratio of diffuse-object holograms recorded in bleached silver halide emulsion depend largely on the reference-to-object beam ratio. When the beam ratio increases, the diffraction efficiency decreases and the signal-to-noise ratio increases, and these two holographic parameters behave in this way no matter what type of processing is used. The presence of noise gratings, generated by scattering at recording, was observed in the response of the holograms when no fixation step is used. The effect of these noise gratings is higher when higher beam ratios are used. Finally, the experimental results of this paper give information about possible ways to improve the holographic characteristics of bleached diffuse-object holograms, and these results are also important when holograms for optical storage ${ }^{25}$ or optical interconnections ${ }^{26}$ are recorded.

Part of this work was supported by the Direcció General d'Ensenyaments Universitaris i Investigació of the Generalitat Valenciana, Spain (project GV-1165/93).

\section{References}

1. H. I. Bjelkhagen, Silver Halide Recording Materials for Holography and Their Processing Vol. 66 of Springer Series in Optical Sciences (Springer-Verlag, New York, 1993).

2. R. L. Lamberts and C. N. Kurtz, "Reversal bleaching for low flare light in holograms," Appl. Opt. 10, 1342-1347 (1971).

3. R. L. Lamberts, "Characterization of a bleached photographic material,” Appl. Opt. 11, 33-41 (1972).

4. N. J. Phillips, A. A. Ward, R. Cullen, and D. Porter, "Advances in holographic bleaches," Photogr. Sci. Eng. 24, 120 (1980).

5. R. R. A. Syms and L. Solymar, "Planar volume phase holograms formed in bleached photographic emulsions," Appl. Opt. 22, 1479-1496 (1983).
6. R. L. van Renesse and F. A. J. Boats, "Efficiency of bleaching agents for holography," Optik 38, 156-168 (1973).

7. P. Hariharan and C. M. Chidley, "Rehalogenating bleaches for photographic phase holograms: the influence of halide type and concentration on diffraction efficiency and scattering," Appl. Opt. 26, 3895-3898 (1987).

8. A. A. Ward and L. Solymar, "Diffraction efficiency limitations of holograms recorded in silver-halide emulsions," Appl. Opt. 28, 1850-1855 (1989).

9. K. Biedermann, "The scattered flux spectrum of photographic materials for holography," Optik 31, 367-389 (1970).

10. R. L. van Renesse, "Scattering properties of fine-grained bleached emulsions," Photogr. Sci. Eng. 24, 114-119 (1980).

11. J. Upatnieks and C. D. Leonard, "Efficiency and image contrast of dielectric holograms," J. Opt. Soc. Am. 60, 297-305 (1970).

12. J. Upatnieks and C. D. Leonard, "Characteristic of dielectric holograms,” IBM J. Res. Devel. 14, 527-532 (1970).

13. H. Kogelnik, "Coupled wave theory for thick hologram gratings," Bell Syst. Tech. J. 48, 2909-2947 (1969).

14. A. Fimia, R. Fuentes and A. Beléndez, "Noise gratings in bleached silver halide diffuse-object holograms," Opt. Lett. 19, 1243-1245 (1994).

15. A. Fimia, L. Carretero, R. Fuentes, and A. Beléndez, "Some remarks on noise sources in silver halide volume holograms," Opt. Eng. 34, 1108-1115 (1995).

16. A. Fimia, A. Beléndez, L. Carretero, and R. Fuentes, "The influence of the photochemical process on the signal-to-noise ratio of diffuse-object holograms," Optik 100, 109-112 (1995).

17. R. K. Kostuk and J. W. Goodman, "Refractive index modulation mechanism in bleached silver halide holograms," Appl. Opt. 30, 369-371 (1991).

18. P. Hariharan, "Rehalogenating bleaches for photographic phase holograms. 3. Mechanism of material transfer,"Appl. Opt. 29, 2983-2985 (1990).

19. R. K. Kostuk and G. T. Sincerbox, "Polarization sensitivity of noise gratings recorded in silver halide volume holograms," Appl. Opt. 27, 2993-2998 (1988).

20. R. K. Kostuk, "Factorial optimization of bleach constituents for silver halide holograms," Appl. Opt. 30, 1611-1616 (1991).

21. A. Beléndez, L. Carretero, and A. Fimia, "Experimental evaluation of shearing effects in volume holograms formed in bleached photographic emulsions," Opt. Laser Technol. 26, 341-349 (1994).

22. A. Beléndez, I. Pascual, R. Fuentes, and A. Fimia, "Calculation of shear angles in holographic gratings recorded in bleached silver halide emulsions," Appl. Phys. B 59, 553-561 (1994).

23. R. R. A. Syms, Practical Volume Holography (Clarendon, Oxford, 1990), Chap. 8.

24. L. Solymar and G. D. G. Riddy, "Noise gratings for single and double beam exposures in silver halide emulsions," J. Opt. Soc. Am. A 7, 2107-2108 (1990).

25. H. Nomura and T. Okoshi, "Storage density limitation of a volume-type hologram memory: theory," Appl. Opt. 15, 550$555(1976)$.

26. R. K. Kostuk, J. W. Goodman, and L. Hesselink, "Optical imaging applied to microelectronic chip-to-chip interconections," Appl. Opt. 26, 3947-3953 (1987). 